\title{
The effects of splenic irradiation on lymphocyte subpopulations in chronic B-lymphocytic leukemia
}

\author{
L. W. M. M. Terstappen'1, B. G. de Grooth', \\ W. van Berkel2, C. H. H. Ten Napel², \\ M. van Reijn² \& J. Greve'
}

1Twente University of Technology, Department of Applied Physics, Cell Characterization Group, Enschede, and 2 Ziekenzorg Hospital, Department of Internal Medicine and Radiotherapy, Enschede, The Netherlands

\begin{abstract}
We describe the effect of splenic irradiation (SI) (0,5-1 Gy weekly) on lymphocyte subpopulations for 7 patients with progressive B chronic B-lymphocytic leukemia (BCLL). Using specific cellular characteristics we could distinguish normal from abnormal cells. The irradiation resulted in a decrease of lymph node size, reduction in spleen volume and decrease in peripheral blood lymphocytes. The one exception was a patient with a prolymphocytoid transformation of B-CLL. For 3 patients SI had to be interrupted or stopped because of severe cytopenia. Quantitation of malignant B cells and normal $T$ lymphocytes revealed that the total irradiation dose which resulted in a specific decrease of malignant lymphocytes varied from patient to patient. Normal T-cell subpopulations, which were increased before SI, decreased to normal or abnormally low values during SI. In previously untreated patients, natural killer (NK) cell numbers decreased more rapidly than $T$-cell subpopulations. For 2 patients refractory to chemotherapy an increase of NK cells was observed upon SI.
\end{abstract}

Accepted for publication June 20, 1988

In chronic B-lymphocytic leukemia (B-CLL), irradiation of thymus, total bone marrow or spleen has been suggested as an effective alternative to chemotherapy and glucocorticosteroid treatment $(1,2)$. Of the various irradiation procedures, splenic irratiation (SI) has resulted in the best reduction of tumor load (2). No serious side effects of SI have been reported. Even with low irradiation doses, SI results in the destruction of a large proportion of the malignant cells. It has also been reported that SI results in a normalization of T-lymphocyte subpopulations (3).
Until now, no effort has been made to establish the effect of SI on different lymphocyte subsets, and to determine the dose of irradiation which yields an optimum benefit for the patients without side effects. We decided to compare the effects of SI on both malignant B lymphocytes and on normal T-lymphocyte subpopulations as a function of radiation dose received during the SI course. In this study 7 patients were treated with SI. We have used multiparameter flow cytometry to quantify the occurrence of malignant cells and to distinguish them from normal 
TABLE 1

Clinical details of patients

\begin{tabular}{lccll}
\hline Patient & Sex & Age & stage Rai & Comment \\
\hline 1 & F & 60 & III & slowly progressive disease for 3 yr \\
2 & M & 66 & IV & newly diagnosed \\
3 & M & 47 & IV & newly diagnosed \\
4 & M & 53 & II & newly diagnosed \\
5 & Mewly diagnosed \\
6 & M & 47 & IV & IV yr treated with chemotherapy* (3 COP courses and \\
& 72 & & $\begin{array}{l}\text { chlorambucil as maintenance treatment) progression } \\
\text { of disease under treatment } \\
6 \text { yr treated with chemotherapy* (7 COP courses and } \\
\text { chlorambucil as maintenence treatement) prolympho- } \\
\text { cytoid transformation of the disease }\end{array}$ \\
\hline
\end{tabular}

$\mathbf{F}=$ female; $\mathbf{M}=$ male; $*$ treatment was stopped 1 month prior to SI.

CD3-, CD4-, CD8-, HNK-1- and CD16-positive lymphocytes during irradiation.

\section{Material and methods}

\section{Patients and controls}

7 B-CLL patients were included in this study. The diagnosis was confirmed by the finding of a monoclonal proliferation of $B$ lymphocytes in the peripheral blood and more than $40 \%$ lymphocytes in the bone marrow. Clonality was demonstrated by expression of a single immunoglobulin light chain, either $\lambda$ or $\kappa$, on the surface. One of the patients showed an increased number of prolymphocytoid cells, indicating a prolymphocytoid transformation of B-CLL (4). The main clinical details of the patients are summarized in Table 1. As controls, 30 healthy donors were used whose ages varied from 22 to $85 \mathrm{yr}$.

\section{Splenic irradiation}

The schedule of splenic irradiation was identical to the one reported by McCann et al (2): patients received a weekly dose of $1 \mathrm{~Gy}$ up to a total dose of $10 \mathrm{~Gy}$, except for patient 2 who received a weekly dose of 0.5 Gy up to a total dose of $5 \mathrm{~Gy}$. Radiation was produced with an $8 \mathrm{MV}$ linear accelerator. Administering the irradiation dose took less than 30 seconds. The irradiation field was established on the basis of an abdominal computer tomographic (CT) scan and adapted each wk in accordance with the clinically approximated spleen size. Blood counts were made before each SI in order to determine whether irradiation could be tolerated. Lymphocyte subpopulations were also determined before irradiation.
Spleen volume and abdominal and mediastinal lymph node sizes were determined from CT scans made before and after SI.

\section{Lymphocyte subpopulation determination}

Human blood was collected by venipuncture into vacutainer tubes containing heparin as anticoagulant. Lymphocytes were obtained after density separation as described in detail elsewhere (5). Because of the low frequency of $\mathrm{T}$ lymphocytes in B-CLL patients, only direct immunofluorescence staining was used with fluorescently labeled monoclonal antibodies. This procedure allows detection of lymphocyte subpopulations by flow cytometry even if they occur in a frequency of only $0.02 \%$ (6). The following monoclonal antibodies were used: Fluorescein (FITC)-labeled monoclonal antibodies: CD3 (anti-leu4, Becton Dickinson, Amersfoort, The Netherlands*); CD4 (anti-leu3*), CD16 (anti-leu11*) and HNK-1 (antileu7*); Phycoerythrin (PE)-labeled monoclonal antibodies: CD8 (anti-leu2a*) and CD5 (anti-leu1*). Lymphocyte analysis was carried out the same day irradiation was performed using the procedure as described in detail elsewhere (7).

Flow cytometric measurements were performed under reproducible apparatus conditions by adjusting the flow cytometer with standard microspheres. This is imperative to obtain reliable information on the amount of cell receptors detected with fluorescently labeled mabs.

Residual monocytes in the preparation were excluded from the results by discrimination on forward and orthogonal light scattering in the flow cytometer (7). 


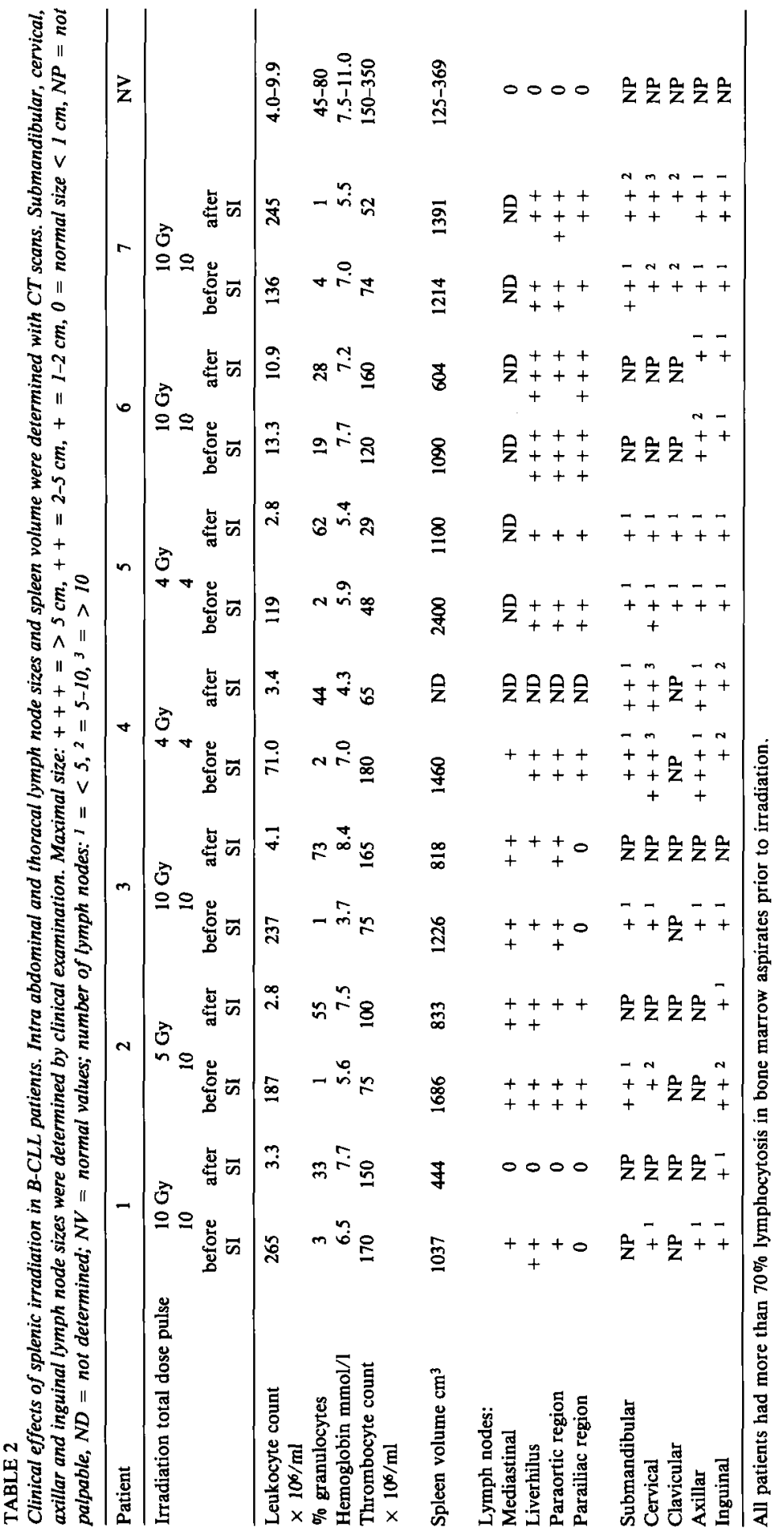



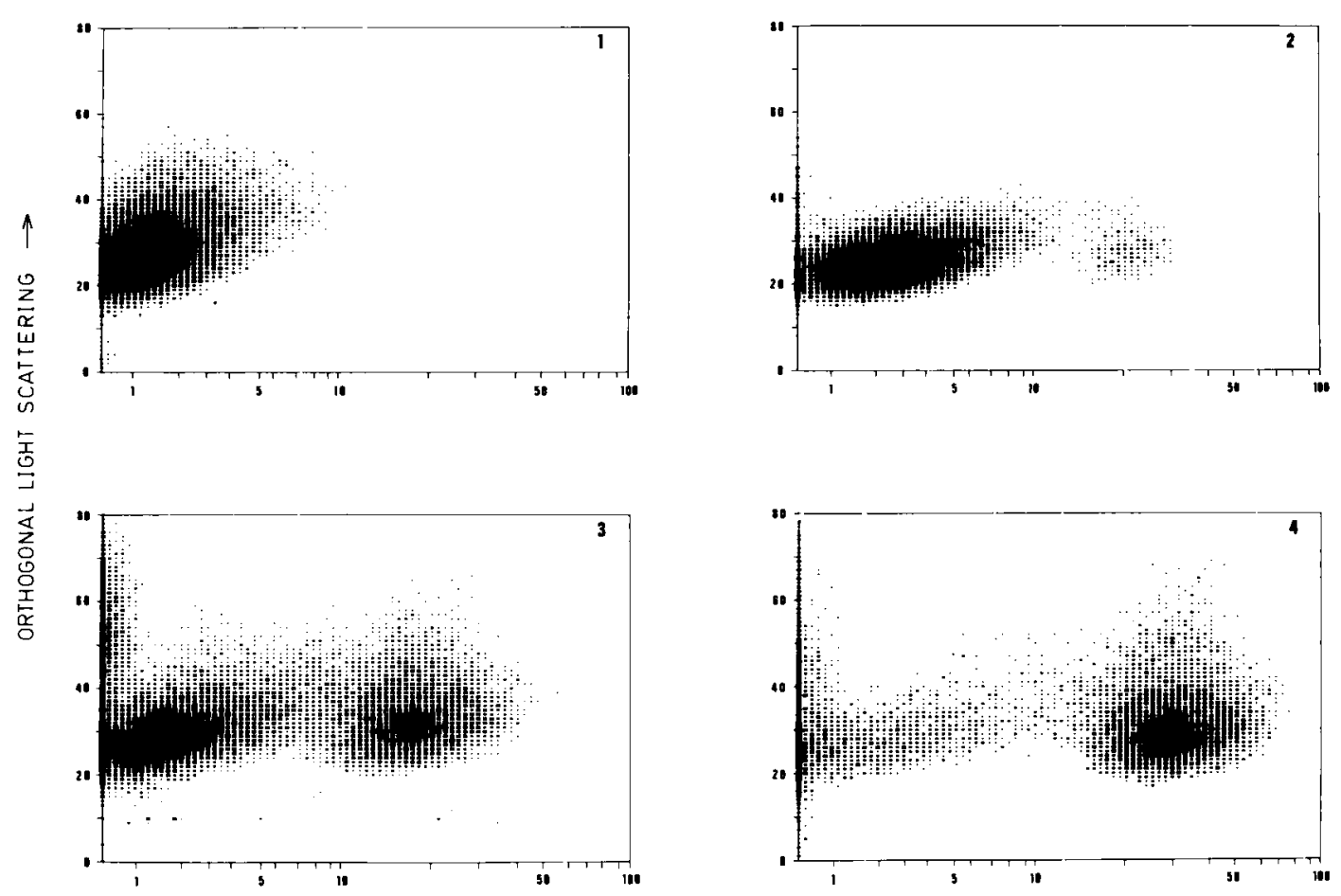

FLUORESCENCE $($ CD $5-P E) \rightarrow$

Figure 1. Two-parameter density map of orthogonal light scattering intensity versus the logarithm of fluorescence intensity of Phycoerythrin-labeled CD5, for the lymphocytes of patient 1 measured before SI (Figure 1.1) and after 3 (1.2), 6 (1.3) and 12 (1.4) wk; SI dose respectively $0,3,6$, and $10 \mathrm{~Gy}$. Equal numbers of cells were represented in the density maps.

\section{Results}

\section{Radiation effects on blood counts and tumor load}

The effect of SI on total white blood cell count, differential granulocyte count, platelet count and blood hemoglobin for all patients is summarized in Table 2 . In the 5 patients not treated previously with chemotherapy the total leukocyte count dropped very rapidly to normal or subnormal values. In 2 patients refractory to chemotherapy the total leukocyte count did not change significantly, or showed an increase. The effect of SI on platelet count and hemoglobin varied considerably from patient to patient. In some patients no change was observed, in others an increase or decrease was found. Only 4 of the 7 patients completed the SI course without complications. In the other patients the SI course had to be interrupted or stopped because of an unacceptable decrease of either platelet count or hemoglobin. None of the patients had gastrointestinal side effects due to SI.

Dimensions of mediastinal lymph node and lymph nodes at the liverhilus, paraaortic and parailiac regions were determined from CT scans (Table 2). Submandibular, cervical, supraclavicular, axillar and inguinallymphnode number and dimensions were determined by clinical examination (Table 2). The decrease in lymphnode dimensions resulting from SI varied from patient to patient. In patient 7 an increase of lymph node sizes was observed.

\section{Discrimination of $T$ cells and malignant $B$ cells}

The receptors for the monoclonal antibody CD5 are present on T cells and malignant B cells of B-CLL 

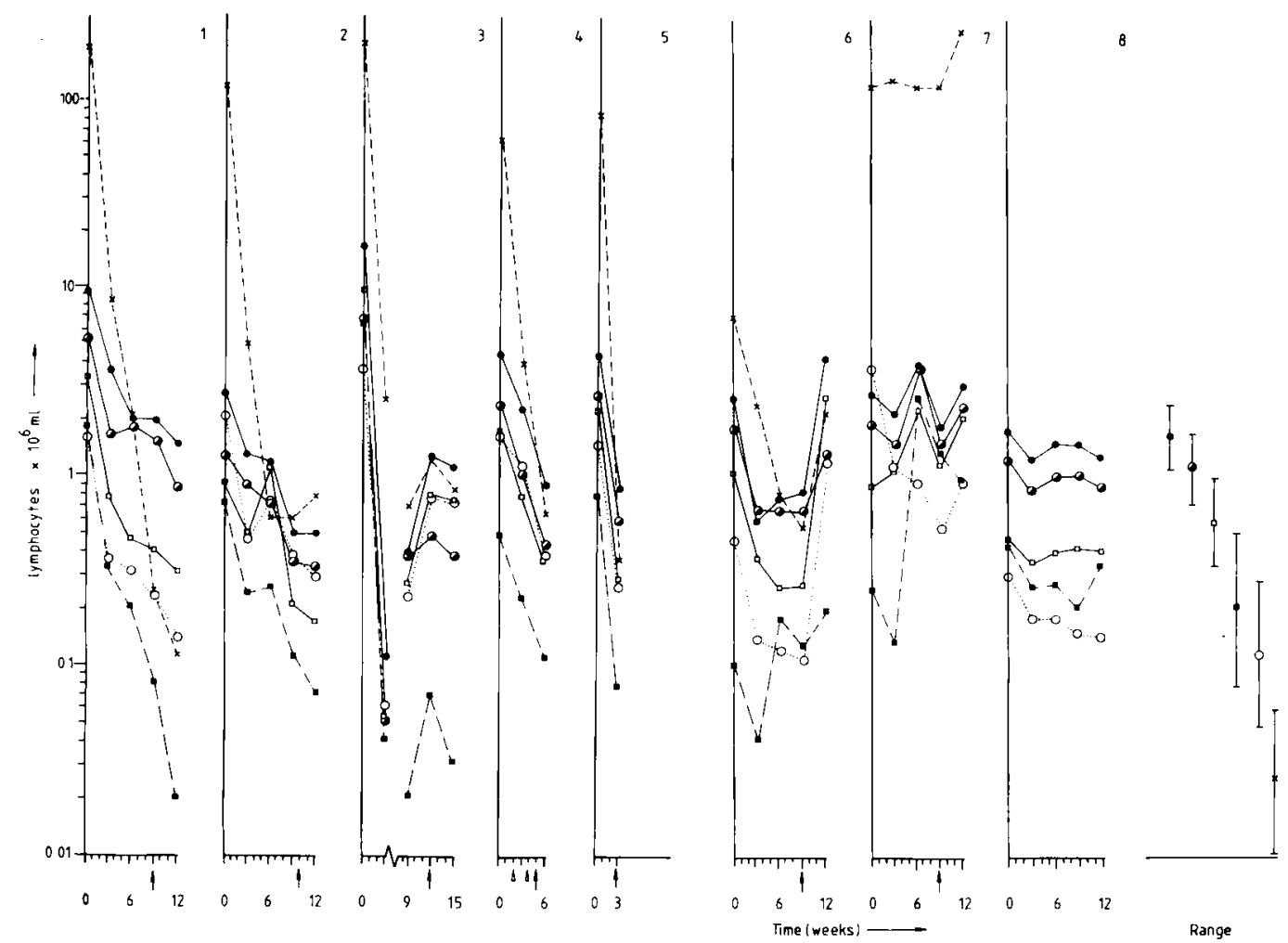

Figure 2. Absolute number of lymphocyte subpopulations determined before SI, before the 4th, 7th and 10th SI and 2 wk after the 10 th (last) SI, unless otherwise indicated. Results are given for patient 1 to 7 in respectively Figure 2.1 to 2.7 . In 2.8 lymphocyte subpopulations of 1 healthy individual followed during the same time period and the range of the absolute number of lymphocyte subpopulations as determined for $\mathbf{3 0}$ healthy individuals are given. Note the logarithmical scale. Explanation of the symbols:

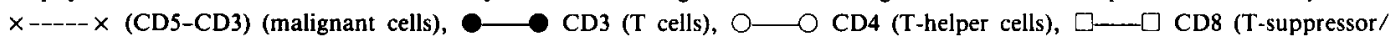
cytotoxic cells), $\square-\ldots-\square \mathrm{CD} 16$ (NK cells), $\bigcirc \ldots . . \circ$ HNK-1 (subset NK cells and cytotoxic T cells), $\downarrow-$ interruption of SI, $\Delta$ no SI, $\uparrow$ last SI.

patients $(8,9,10,11)$. We have found that the CD5 receptor density differs strongly for both populations: the mean CD5 fluorescence signal of $\mathrm{T}$ cells is more than $\mathbf{1 0}$ times as large as that of the malignant $B$ cells. This was found for 15 B-CLL patients investigated in our laboratory. Using this observation we could determine the differential effect of SI on $T$ cells and malignant $B$ cells. This is illustrated in Figure 1, where two-parameter density maps of orthogonal light scattering intensity versus the logarithm of the fluorescence intensity of CD5 are given for patient 1 . Determinations were made before SI and after 3, 6 and 10 Gy had been received (Figure 1.1 to 1.4 respectively, each of which contains data from an equal number of cells). In Figure 1.1 the majority of lymphocytes showed a relative low fluorescence intensity, a property of malignant $B$ cells. In Figure 1.2 a second population with a high fluorescence intensity could clearly be seen. These were $T$ cells which possess high densities of CD5 receptors. Figures 1.3 and 1.4 show a progressive increase of the relative number of $T$ cells. Malignant B cells were almost absent after the irradiation course was completed (Figure 1.4). This Figure clearly illustrates the selective lethal effect of SI on malignant $B$ cells. 

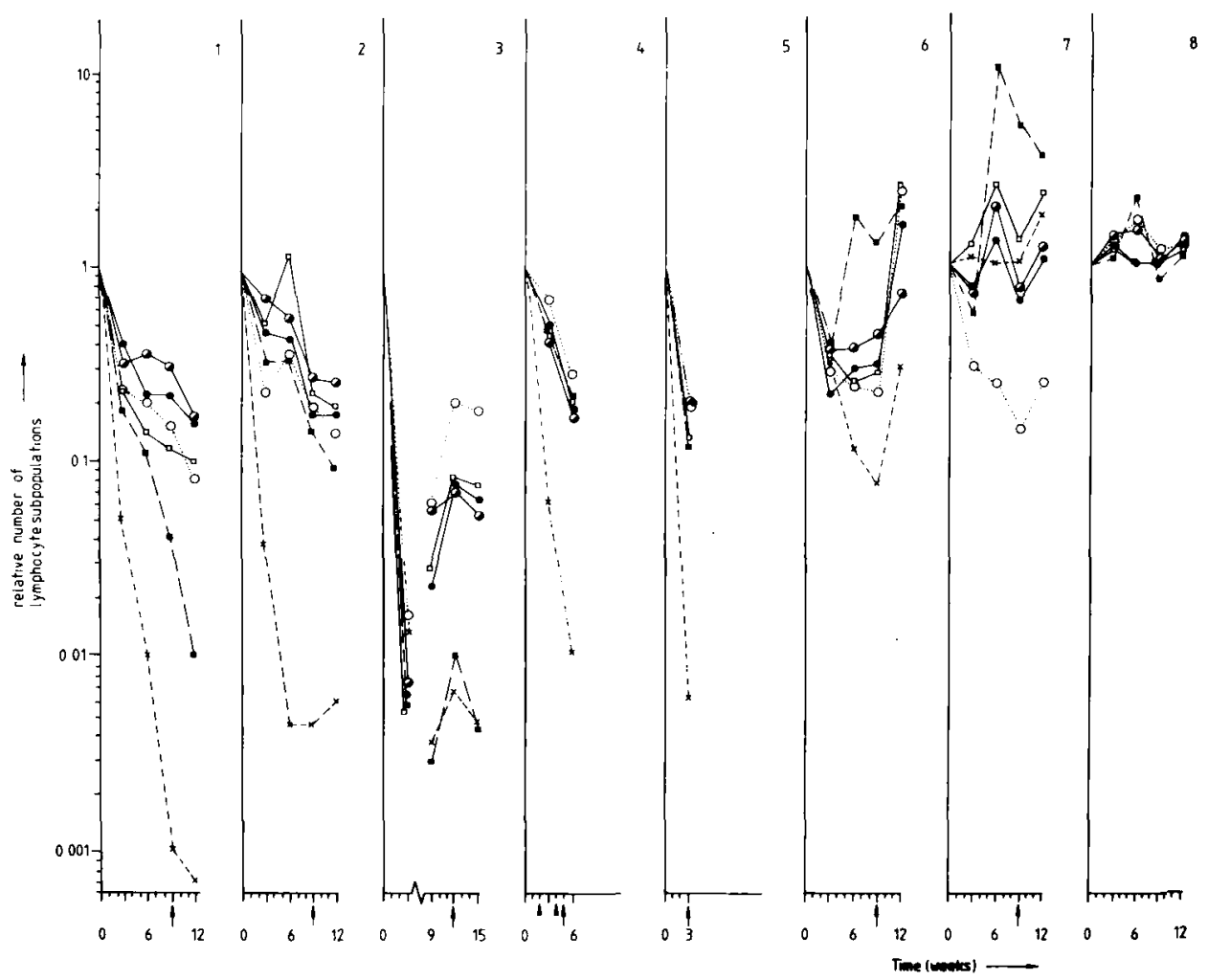

Figure 3. Relative sensitivity to SI of the different lymphocyte subpopulations. Occurrence of the absolute number of lymphocyte subpopulations during and after the SI (before 4th, 7th, 10th and $2 \mathrm{wk}$ after the 10th SI) were compared with the absolute number of lymphocytes before SI. Results are given for patient 1 to 7 in Figure 3.1 to 3.7 respectively. In 3.8 data are given for 1 healthy individual followed during an equal time period. Note the logarithmical scale. Explanation of the symbols

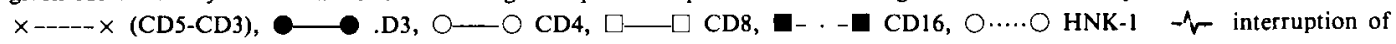
SI, $\triangle$ no SI, $\uparrow$ last SI.

\section{Quantitation of}

\section{lymphocyte subpopulations}

The absolute numbers of different lymphocyte subpopulations were detemined immediately before 1st, 4th, 7th, 10th SI and 2 wk after the last SI. Since $T$ cells and malignant $B$ cells were positive with the monoclonal antibody CD5 and the receptors for the monoclonal antibody $\mathrm{CD} 3$ occurred only on $T$ cells, the percentage of malignant lymphocytes was defined as the percentage of CD5-positive cells minus the percentage of CD3positive lymphocytes. This was not completely correct since immature $T$ cells and a small percentage of normal $B$ cells were also positive with CD5 and negative with CD3. However, the occurrence of these cells in normal individuals was very low (less than $0.07 \times 10^{6} \mathrm{ml}$ ).

In Figure 2.1 to 2.7 absolute numbers of different lymphocyte subpopulations are presented for all patients. For comparison, we followed the different lymphocyte subpopulations for 1 healthy individual during a period equal to the irradiation course given to the patients. We also determined the normal range of lymphocyte subpopulations for 30 healthy donors. These data are given in Figure 2.8.

In all patients, except patient 7 , the malignant $B$ cells decreased very rapidly following the initiation of the irradiation therapy. The absolute effect of SI on the malignant cells varied significantly 
between patients. Normal values of $\mathrm{CD}^{+} \mathrm{B}$ lymphocytes were not reached in any of the patients.

The absolute numbers of $\mathrm{CD}^{+}, \mathrm{CD}^{+}$and $\mathrm{CD8}^{+}$cells were abnormally high or within normal values for the 5 patients who had not been treated by chemotherapy prior to SI. These numbers fell to the normal or subnormal range during the course of the SI. In contrast, the $\mathrm{CD}^{+}, \mathrm{CD}^{+}$and $\mathrm{CD} 8^{+}$lymphocytes in patient 7 (previously treated by chemotherapy) were unaffected by SI. There was a decrease in number of $\mathrm{CD}^{+}, \mathrm{CD}^{+}$and $\mathrm{CD}^{+}+$lymphocytes in patient 6 during SI, followed by a rapid increase immediately after SI.

In all patients the number of HNK-1 + lymphocytes was elevated before SI. During irradiation the number of HNK-1 + lymphocytes decreased in the patients. However, in patient 6 the number of HNK $-1^{+}$cells increased rapidly after the SI course, as was observed for the $T$ lymphocytes.

The numbers of CD16-positive lymphocytes (NK cells) determined before SI were increased in patients 1, 2, 3 and 5 (Figure 2.1, 2.2, 2.3, 2.5) and fell within normal range for patients 4,6 and 7 (Figure 2.4, 2.6, 2.7). During the irradiation course the NK cells dropped below normal values except for the patients refractory to chemotherapy (6 and 7, Figure 2.6, 7) for whom an increase of NK cells was observed.

A remarkable observation during the SI course of patient 3 (Figure 2.3) was that both malignant cells and normal lymphocyte subpopulations dropped to abnormally low values. For this patient the course was interrupted because of severe fever for which no source could be found. After a delay of $5 \mathrm{wk}$, the SI was continued. At that time, the lymphocyte subpopulations were increased to almost normal values, except for the NK cells which remained very low. The subsequent SI did not significantly change this pattern.

\section{Relative sensitivity of the different lymphocyte subpopulations to splenic irradiation (SI)}

The relative sensitivity of the different subpopulations to SI in the 7 patients is presented in Figure 3.1 to 3.7. For comparisons the variation of the different lymphocyte subpopulations is given for a healthy individual during an equivalent time period (Figure 3.8). It was clearly demonstrated that the malignant cells were the cells most sensitive to SI (Figure 3.1-3.5) in previously untreated patients: a decrease by a factor of 100 to more than 1000 was observed.

For patients refractory to chemotherapy, the effect of irradiation was less selective. In patient 6 (Figure 3.6) the malignant cells decreased by a factor of 20 as compared with normal lymphocytes. In patient 7 (Figure 3.7) there was no effect on either the malignant or the $T$ cells.

A striking reduction in the NK cell fraction was observed in patients 1 and 3 (Figure 3.1, 3.3). This fraction was 10 times more sensitive to SI than the CD3-positive $T$ cells. In patient 2 and 5 (Figure 3.4) no difference between NK cells and T cells was found. Previously treated patients (6, 7, Figure 3.6, 3.7) showed an increase of NK cells and a decrease of HNK-1 ${ }^{+}$lymphocytes during treatment.

Differences in sensitivity of the $\mathrm{CD}^{+}, \mathrm{CD}^{+}$, $\mathrm{CD}^{+}$and $\mathrm{HNK} 1+$ cells were significant, but no consistent pattern could be discerned in the patients studied.

\section{Discussion}

In this study we evaluated the effect of splenic irradiation (SI) on lymphocyte subpopulations of 7 patients with B-CLL. In agreement with previous studies of Aabo et al (1), Byhardt et al (12) and McCann et al (3), we showed that, in all previously untreated patients, SI resulted in a drastic decrease in the number of lymphocytes. In one patient with a malignant prolymphocytoid transformation of the B-CLL, an increase of tumor load in lymph node, spleen and peripheral blood was observed in spite of SI.

The massive lymphocyte loss resulting from SI is not fully understood. The decrease of spleen size could be explained by the recognized destructive effect of irradiation on B cells (13, 14). To explain the enormous decrease in the number of peripheral blood lymphocytes and lymph node sizes, the splenic lymphocyte pool of the patients has to be larger as compared 
with the peripheral lymphocyte pool (15). Furthermore, the lymphocyte traffic from lymph nodes and peripheral blood to the spleen has to be considerable.

The effect of SI on normal hematopoiesis varied significantly between the patients. Hemoglobin and platelet counts were seen to increase, to decrease, or to remain constant in the patients who received SI. In only 4 of the 7 patients could the entire SI course be given. These observations clearly showed that SI was not always as free from side effects as was reported in other studies $(1,2,3,12,16)$. In those studies a comparable patient population and irradiation scheme was used.

The effect of SI on lymphocyte counts also varied between the patients (Figures 2 and 3 ). The observed differences in the effect of SI on malignant $B$ cells between the patients could be explained by different maturation stages of the malignant cells, since the radiosensitivity of $B$ cells is dependent on their differentiation stage $(11,17)$.

The decrease of malignant cells was considerably larger than the decrease of normal $T$ cells (Figure 3), which could be explained by the greater radiosensitivity of B cells compared with $T$ cells (18). The absolute numbers of cells of the different $T$-cell subpopulations which were above average before SI decreased during SI to lower than normal values for most patients. This could disrupt the reactivity of the immune system and therefore make patients more susceptible to infection.

Patient 2 received a weekly SI dose of 0.5 Gy, half the SI dose administered to the other patients. Nevertheless, the rate at which malignant cells decreased was at least as fast as in the other patients. The sensitivity of the $T$ cells to SI in this patient was less than in the other patients. This suggests that it may be worthwhile to determine the minimal SI dose to cause a decrease of malignant $B$ cells with minimum effects on the $T$ cells.

At first sight the different $T$-cell subpopulations of an individual patient showed a similar response to the SI. Although a closer examina- tion revealed significant changes in CD4/CD8 ratios during the irradiation course, we did not observe consistent changes in the CD4/CD8 ratios. This was in contrast with the results of McCann et al (3) who found a normalization of $T \mu / T \gamma$ ratios after SI of B-CLL patients. The variation observed in our data indicates that one should be careful not to draw conclusions based upon a single determination.

The initial sensitivity of the different $T$-cell subpopulations to SI was comparable for 5 of the 7 patients (Figures 3.1, 3.2, 3.4, 3.5, 3.6). However, the observation that in patient 3 (Figure 3.3) this sensitivity was initially more than 40 times greater than in the other patients, whereas in patient 7 (Fig. 3.7) there was no change in $\mathrm{T}$-cell subpopulations at all during SI, suggests that not only the radiosensitivity of the $T$ cells plays a role in the response of $T$-cell subpopulations to SI. Possible explanations for the difference in sensitivity to SI may be:

1. The relative occurrence of T-cell subpopulations in the spleen of patient 3 was much greater than in the other patients, whereas in patient 7 relatively few $T$ cells were within the spleen.

2. Radiosensitivity of the $T$ cells of patient 3 was greater and of patient 7 was less than in the other patients.

3. Immunoregulation of homing and proliferation of $\mathrm{T}$ cells which respond to changes in the number of malignant cells.

The latter explanation may be the most likely one since in patient 7 no response to the number of malignant $B$ cells nor to the number of $T$ cells was observed upon SI.

Several authors have described a relative or absolute increase of CD8 lymphocytes in B-CLL patients resulting in a decreased CD4/ CD8 ratio $(6,19,20,21,22,23,24)$. The increase of $\mathrm{CD} 8$ lymphocytes is often ascribed to an increase of the number of CD8 suppressor lymphocytes. We have reported that in B-CLL patients these CD8 lymphocytes mainly consist of cytotoxic lymphocytes $(5,6)$. In the 
latter study, the hypothesis was put forward that these cytotoxic lymphocytes were directed specifically against the leukemic cells. This might explain the spontaneous remissions sometimes observed in untreated B-CLL patients (25). In this study we found that the absolute and relative amounts of cytotoxic lymphocytes were abnormally high in all patients before SI, supporting the importance of cytotoxic lymphocytes in B-CLL patients. Especially the numbers of HNK-1 lymphocytes were very high, indicating that the cytotoxic lymphocytes mainly consist of cytotoxic $\mathrm{T}$ cells and not NK cells. This was in accordance with the studies of Foa et al $(26,27)$, although in the latter study a decrease of NK cells as compared with healthy donors was found. We did not observe such a decrease in our patients.

A remarkable observation was that the NK cells identified with $C D 16$ were more sensitive to SI than CD3-, CD4-, CD8- and HNK-1-positive lymphocytes in patients not treated prior to SI. When NK cells do play an active role in the natural defence against leukemic cells, a major part of this defence is destroyed because of the selective disappearance of NK cells through SI. Patients refractory to chemotherapy showed an opposite effect of the SI on cytotoxic lymphocyte subpopulations, namely an increased number of NK cells (CD16) and a decreased number of HNK-1 lymphocytes (identifying a subset of NK cells and T cell-dependent cytotoxic lymphocytes). This suggests that the decrease found in patients not treated prior to SI was not caused by a greater radiosensitivity of NK cells, but that immunoregulatory processes played the major role.

\section{Acknowledgments}

We are very grateful to Y. Kraan and I. SegersNolten for skilful laboratory work, to Dr. R. Holdrinet (Radbout Hospital, Nijmegen, The Netherland) and Dr. G. S. Hoogendoorn (St. Geertruiden Hospital, Deventer, The Netherlands) for allowing us to study their patients and to Dr. M. R. Loken for critical reading of the manuscript.

\section{References}

1. Aabo K, Walbom-Jørgensen $S$. Spleen irradiation in Chronic Lymphocytic Leukemia (CLL): Palliation in patients unfit for splenetomy. Am J Hematol 1985;19:17780.

2. Paule B, Cosset JM, Le Bourgeois JP. The possible role of radiotherapy in chronic Lymphocytic Leukaemia: A critical review. Radiotherapy Oncol 1985;4:45-54.

3. McCann SR, Whelan CA, Breslin B, Temperley IJ. Lymphocyte subpopulations following splenic irradiation in patients with chronic Lymphocytic Leukaemia. Br J Haematol 1982;50:225-9.

4. Enno A, Catovsky D, O'Brien M, Cherchi M, Kumaran TO, Galton DAG. Prolymphocytoid transformation of chronic lymphocytic leukaemia. Br J Haematol 1979;41:918.

5. Terstappen LWMM, de Grooth BG, Nolten GMJ, ten Napel $\mathrm{CHH}$, van Berkel W, Greve J. Physical discrimination between human T-Lymphocyte subpopulations by means of Lightscattering, revealing two populations of T8positive cells. Cytometry 1986;7:178-83.

6. Terstappen LWMM, de Grooth BG, van Berkel $W$, ten Napel CHH, Greve J. Abnormal distribution of CD8 subpopulations in B-Chronic Lymphocytic Leukaemia identified by flowcytometry. Leuk Res (in press).

7. Terstappen LWMM, de Grooth BG, ten Napel CHH, van Berkel W, Greve J. Discrimination of human cytotoxic Lymphocytes from regulatory and B-Lymphocytes by orthogonal Lightscattering. J Immunol Methods 1986; 95:211-6.

8. Boumsell L, Coppen H, Pham P, et al. An antigen shared by human $T$ cell subset and $B$ cells: Distribution on normal and malignant Lymphoid cells. I Exp Med 1980;152:229-34.

9. Martin PJ, Hansen JA, Nowinski RC. Monoclonal antibodies recognizing normal human $T$ Lymphocytes and malignant human B Lymphocytes: A comparative study. J Immunol 1981;127:1920-3.

10. Rovston I, Majda JA, Biard SM, Meserve BL, Griffiths JC. Human $\mathrm{T}$ cell antigens defined by monoclonal antibodies: The 65,000-dalton antigen of $\mathrm{T}$ cells (T65) is also found on chronic Lymphocytic Leukemia cells bearing surface immunoglobulin. J Immunol 1980;125:725-31.

11. Terstappen LWMM, de Grooth BG, ten Napel CHH, van Berkel W, Greve J. Flow cytometric characterization of chronic lymphocytic leukemias using orthogonal light scattering and quantitative immunofluorescence. Blut (in press).

12. Byhardt RW, Brace $\mathrm{KC}$, Wiernik $\mathbf{P H}$. The role of splenic irradiation in chronic Lymphcytic Leukemia. Cancer 1975;35:1621-5.

13. Anderson RE, Warner NL. Ionising radiation and the immune respon se. Adv Immunol 1976;24:215-67.

14. Johnson RE. Radiotherapy as primary treatment for chronic Lymphocytic Leukaemia. Clin Haematol 1977; 6:237-44.

15. Spivak JL, Perry S. Lymphocyte kinetics in Chronic Lymphocytic Leukaemia. Br J Haematol 1970;18:511-22. 
16. Roncadin M, Arcicasa M, Trovó MG, et al. Splenic irradiation in chronic lymphocytic leukaemia. A 10-year experience at a single institution. Cancer 1987;60:2624-8.

17. Vann DC, Makinodan T. In vitro antibody synthesis by diffusion chamber culture of spleen cells. J Immunol 1969;102:442-50.

18. Durum SK, Gengozian N. The comparative radio sensitivity of $T$ and $B$ lymphocytes. Intern $J$ Radiat 1978; 34:1-15.

19. Catovsky D, Lauria F, Matutes E, et al. Increase in $T \gamma$ lymphocytes in B cell chronic lymphocytic leukaemia. $\mathrm{Br} \mathrm{J}$ Haematol 1981;47:539-44.

20. Herrman F, Lochner A, Philippen H, Jauer B, Ruhl H. Inbalance of $\mathrm{T}$-cell subpopulations in patients with chronic lymphocytic leukaemia of the B cell type. Clin Exp Immunol 1982;49:157-62.

21. Markey GM, Alexander HD, Agnew AND, et al. Enumeration of absolute numbers of $T$ Lymphocytes subsets in B-chronic lymphocytic Leukaemia using an immunoperoxidase technique: Relation to clinical stage. $\mathrm{Br} \mathrm{J}$ Haematol 1986;62:257-73.

22. Mills KHG, Cawley JC. Suppressor T cells in B-cell chronic lymphocytic leukaemia: Relation to clinical stage. Leuk Res 1982;6:653-7.

23. Mittelman A, Denny T, Gebhard C,M Cirrincione $C$, Kurland E, Kozinek B. Analysis of T cell subsets in B cell chronic lymphocytic leukemia: A correlation with the stage of disease. Am J Hematol 1984;16:67-73.
24. Platsoucas CD, Galinski M, Kempin S, Reich L, Clarkson B, Good RA. Abnormal T lymphocyte subpopulations in patients with B-cell chronic lymphocytic leukemia: an analysis by monoclonal antibodies. J Immunol 1982;129:230512.

25. Horning SJ, Rosenberg SA. The natural history of initially untreated low-grade non-Hodgkin's Lymphomas. N Engl J Med 1984;311:1471-5.

26. Foa R, Fierro MT, Lusso P, et al. Reduced Natural Killer T-cells in B-cell chronic Lymphocytic Leukaemia identified by three monoclonal antibodies: Leu11, A10, AB8.28. Br J Haematol 1986;62:151-4.

27. Foa $R$, Lauria F, Lusso $P$, et al. Discrepancy between phenotypic and functional features of natural killer T-Lymphocytes in B-cell chronic Lymphocytic Leukaemia. Br J Haematol 1984;509-16.

Correspondence to:

J. Greve

University of Twente

Dept. of Applied Physics

Cell Characterization Group

P.O. Box 217

7500 AE Enschede

The Netherlands 